\title{
Arbitrarily Large Neutron Amplification in Subcritical Nuclear Reactors
}

\author{
Antoine Tilloy $\odot^{*}$ \\ Max-Planck-Institut für Quantenoptik, Hans-Kopfermann-Straße 1, Garching 85748, Germany
}

(Received 13 December 2020; revised 11 June 2021; accepted 22 June 2021; published 26 July 2021)

\begin{abstract}
In a subcritical reactor, each neutron produces only $k_{\text {eff }}<1$ neutrons per generation (asymptotically and on average), and thus, the neutron population decreases exponentially in the absence of an external source. The chain reaction is thus easy to stop, making such reactors inherently stable. Interestingly, and contrary to common wisdom, there is no relation between $k_{\text {eff }}$, the external source intensity, and the output power of the reactor. Here, various possible strategies to exploit this fact are presented, and they are applied to the design of a rudimentary multilayer system that allows an arbitrarily large number of fissions per source neutron to be reached, while keeping $k_{\text {eff }}<1$ fixed (here, $k_{\text {eff }}=0.97$ ). The behavior of the amplifier is verified with simple Monte-Carlo transport simulations. The proposal admittedly brings complications and subtleties that need to be studied further, but, if developed, could allow subcritical reactors to be driven with faint neutron sources, e.g., radioisotope based.
\end{abstract}

DOI: 10.1103/PhysRevApplied.16.014059

\section{INTRODUCTION}

Subcritical nuclear reactors are serious candidates for the incineration of minor actinides produced by current light-water reactors. With an asymptotic neutron multiplication, $k_{\text {eff }}<1$, they allow an intrinsically stable operation without the need for delayed neutrons and can thus operate with a much higher minor actinide content than more standard critical reactors. However, neutrons have to come from somewhere, and one [1] of the drawbacks of such designs lies in the need for an efficient external neutron source. Current proposals use a spallation source driven by a high-current proton accelerator. To reach an industrially meaningful actinide incineration or electricity production, the accelerator power required goes well beyond what is available off-the-shelf.

The high power need comes from the requirement of a $k_{\text {eff }}$ value that is not too close to 1.0 , to ensure safe operation; the choice of $k_{\text {eff }} \simeq 0.95$ is usually made in the literature [2]. At an intuitive level, this suggests that one neutron can produce, on average, 0.95 neutrons per generation, and thus, a number of fissions in the order of 20 from the first fission induced by the spallation source. While this rule of thumb may give a correct order of magnitude for standard accelerator-driven subcritical reactor (ADSR) designs and explains the need for a high-intensity neutron

*antoine.tilloy@gmail.com

Published by the American Physical Society under the terms of the Creative Commons Attribution 4.0 International license. Further distribution of this work must maintain attribution to the author(s) and the published article's title, journal citation, and DOI. Open access publication funded by the Max Planck Society. source, it can give the wrong order of magnitude in more complicated situations.

We need to keep track of neutron generations and consider the fact that the multiplication per neutron, $k_{i}$, can be generation dependent because of the geometry of the system. The number of neutrons produced from a singlesource neutron is, on average,

$$
N=k_{1}+k_{1} k_{2}+k_{1} k_{2} k_{3}+\cdots=\sum_{i=1}^{+\infty} \prod_{j=1}^{i} k_{j} \stackrel{\text { def. }}{=} \frac{1}{1-k_{s}} .
$$

This $N$, or equivalently $k_{s}$, is what we focus on when we consider the total neutron multiplication of the system and the output power for a given source. $k_{s}$ is the equivalent multiplication we would need to get the same $N$ had the multiplication $k_{i}$ been generation independent. Importantly, it is unrelated to $k_{\text {eff }}$, which is defined as

$$
k_{\text {eff }} \stackrel{\text { def. }}{=} \lim _{i \rightarrow+\infty} k_{i}
$$

which is the asymptotic multiplication. Only $k_{\text {eff }}$ governs the long-term reactor kinetics and is thus relevant to assess criticality. We see that, in principle, there is no theoretical obstacle to an arbitrarily large $N\left(k_{s}\right.$ arbitrarily close to 1.0$)$ with a fixed subcritical $k_{\text {eff }}$, if the initial $k_{i}$ values are large.

In fact, some ADSR designs obtain modest amplification from the naive $k_{\text {eff }}=k_{s}$ relation, with a highly enriched core near the spallation source surrounded by low-enriched blankets. However, such designs typically reduce the accelerator requirements only by a moderate amount. 
This raises a natural question: is it possible to design a core such that $k_{\text {eff }}$ is fixed but $N$ is large enough to substantially reduce the accelerator requirements? Can multiplication be increased to the point that a much smaller accelerator can be used or even that much weaker radioisotope sources can be used? Is there a physical limit to amplification due to neutron transport in existing materials? Is there a practical limit with current technology and engineering constraints?

\section{STRATEGIES}

To increase $N$, while keeping $k_{\text {eff }}<1$, one needs to create an irreversibility or decoupling in the neutron current. Imagine we are given a perfect neutron diode, that is, a material letting neutrons go in one, and only one, direction. Then, we could easily stack subcritical sections, separating them by the diodes and the total would remain subcritical, with the same $k_{\text {eff. }}$. However, neutron amplification would be enhanced geometrically with the number of layers, without obvious physical limit.

Of course, perfect neutron diodes do not grow on trees, and the challenge lies in creating a genuine unidirectionality of the neutron current, so that the criticality, $k_{\text {eff }}$, does not increase (or only in a subleading way) when stacking more subcritical layers.

There are at least four conceptually different ways to create a one-way neutron current between different parts of a reactor, as summarized in Fig. 1. In practice, these different methods can be combined and are only separated to emphasize the physical mechanism at play.

The strategy outlined in Fig. 1(a) corresponds to one that was considered as early as 1957 [3]. The idea is to exploit the fact that certain materials, like cadmium, are almost transparent to fast neutrons, but have a large capture cross section for thermal neutrons. Intuitively, by putting such a thermal absorber and following it with a moderator, one obtains a material that can be (approximately) crossed only one way by neutrons (and converts fast neutrons into slow neutrons in the forward direction).

The second strategy [Fig. 1(b)] is, in a way, opposite to the first. Instead of capturing slow neutrons, one could selectively capture fast neutrons with a material like lowenriched uranium, which works as a neutron multiplier only in the thermal spectrum, while neutron capture by ${ }^{238} \mathrm{U}$ proportionally dominates in a fast spectrum. The idea is to put a moderator first and then this fast-spectrum capture material to obtain a diode. This one-way amplification through thermal fission is likely to be insufficient to create genuine irreversibility alone, but seems to play a role in multiplication achieved by various proposals.

The third option [Fig. 1(c)] is to use a threshold fissile material like ${ }^{237} \mathrm{~Np}$ or ${ }^{241} \mathrm{Am}$, which is only fissile in a fast spectrum and, consequently, multiplies only fast
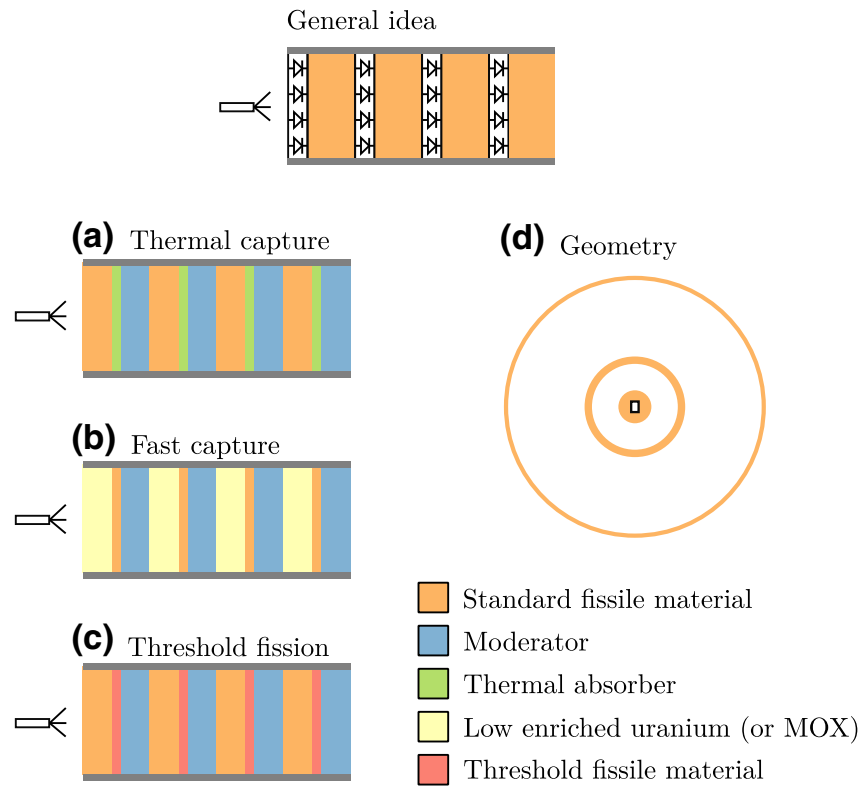

FIG. 1. Strategies for multistage amplification. Subcritical cells separated by ideal neutron diodes act as neutron amplifiers. In practice, this can be implemented with four distinct physical strategies: (a) thermal capture, (b) fast capture, (c) threshold fission, and (d) geometry.

neutrons. As before, this allows a one-way amplifier to be constructed with the addition of a moderator.

The fourth option [Fig. 1(d)] is purely geometric and is, in a way, the simplest (see Ref. [2]). The idea is to decouple stages by having them grow in size (or in surface) as one moves away from the source. This way, neutrons from an earlier stage can reach the following one, where they induce fission, but this fission creates neutrons that have less chance to hit the initial stage. An example consists of considering concentric fissile spheres of quickly growing radii and shrinking width. If the ratio of the radii is large enough, the total $k_{\text {eff }}$ of the set of spheres is the same as that of each of them taken individually. However, the number of fissions is amplified at each layer.

Finally, a more recent idea is to exploit magnetic fields to deflect neutrons via coupling to their spin [4]. As it is far more speculative than the previous strategies outlined, this option is not considered further here.

\section{PREAMPLIFIER EXPLORATION}

The objective of this study is to see how a combination of the previous strategies could fare with physically realistic materials and geometry (including neutron loss). To this end, it seems easier to distinguish between two levels of amplification that would be joined together in a complete reactor, but that come with starkly different constraints. The first stage, the preamplifier, would bring an arbitrarily faint neutron flux to the intensity needed to drive a second 
"power" stage. This second stage, a more standard reactor core, would be typically made of only a few simple layers, with low amplification but high power density.

A proper study of a modestly amplifying reactor core is difficult, but is reported in the literature [5,6]. In fact, such designs are even implemented (at zero power) in the YALINA-Booster experiment at The Joint Institute for Power and Nuclear Research in Belarus [7-9]. There, it is observed that there is still a non-negligible backflow of neutrons through the diode (or valve) [10]. This is important for theoretical modeling: one cannot assume perfect diodes, and one must take into account the complex transport of neutrons through the materials of the reactor.

For few-layer power cores, the main difficulties come from the need to take into account thermohydraulic constraints (which limit the local power density), the evolution of reactivity, neutron economy, and simplicity constraints. Typical two-layer cores would exploit a threshold fissile or highly enriched center in a fast spectrum and a lessenriched moderated outer region. For realistic designs, a modest (fixed) amplification can be achieved, but it is not sufficient to drastically change the neutron-driving requirements.

On the other hand, a many-layer preamplifier with arbitrary amplification has never been properly studied. The constraints are different from those of the core. To achieve massive amplification, the design should be scalable, which a priori excludes investigating a spherical or cylindrical concentric geometry and favors a linear stacking of layers. Further, the power density could be made substantially lower than that in the core, especially in the layers closer to the (faint) source. This, in turn, means that cooling, reactivity evolution, and neutron economy would be secondary constraints. For an initial exploration, it makes sense to focus primarily on neutron transport.

Such a preamplifier might be useful as a standalone device, e.g., to produce intense neutron pulses (at very large instantaneous but low average power), or breed radioisotopes and tritium, which further motivates its study.

Finding an optimal or even adequate design of a preamplifier given reasonable engineering and safety constraints is a nontrivial task. The objective here is more modest: to demonstrate that (arbitrary) amplification is physically feasible, at least, in principle. To this end, every option available, apart from geometry, is used to keep a scalable design, where an arbitrary number of layers can be stacked. More precisely, a stack of layers, each made of four cells containing (1) a fissile material with fission enhanced in the thermal spectrum (a mixed oxide of ${ }^{239} \mathrm{Pu}$ and ${ }^{238} \mathrm{U}$ ), (2) a threshold fissile material (metallic ${ }^{241} \mathrm{Am}$ ), (3) a thermal neutron absorber (cadmium), and (4) a moderator (heavy water), is considered. This combines the three nongeometric amplification methods mentioned in Sec. II. This choice is largely arbitrary, and it is likely that much better

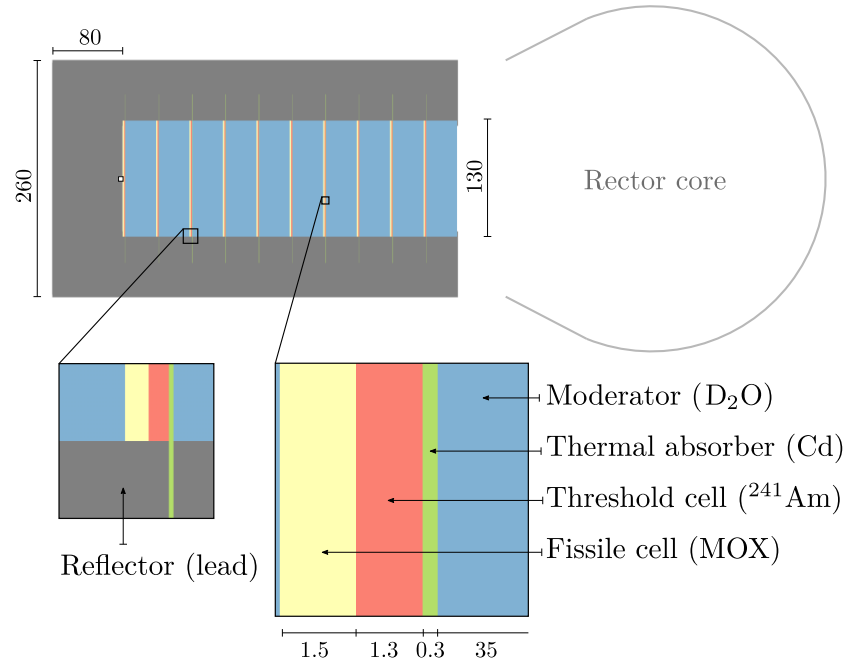

FIG. 2. 10-layer example. Preamplifier is a cylinder with $130 \times 130 \mathrm{~cm}^{2}$ square basis surrounded by a lead reflector. Moderator is heavy water, thermal absorber is cadmium, threshold material is metallic ${ }^{241} \mathrm{Am}$, and fissile cell is mixed oxide (MOX; $43 \%{ }^{239} \mathrm{Pu}$ and $57 \%{ }^{238} \mathrm{U}$ for $\left.k_{\mathrm{eff}}=0.97\right)$. Cadmium layer goes halfway through the reflector to reduce back coupling of thermal neutrons through the reflector. Neutron source is shown by a white dot against the reflector and just before the first layer. Dimensions are shown in $\mathrm{cm}$.

combinations can be found. The precise geometry, including the reflector, is provided in Fig. 2 for a stack of 10 layers.

\section{RESULTS}

A simple test of the previous proposal is carried out with the help of the Monte-Carlo transport simulation tool openMC [11] developed at MIT. The full preamplifier three-dimensional geometry outlined in Fig. 2 -filled with fissile isotopes, moderators, and absorbers mentioned previously - is simulated for various numbers of layers and without additional assumptions.

After some trial and error, but no systematic optimization, it is easy to reach a scalable amplification of about 1.6 per layer (1.6 times more neutron flux and fissions in layer $n+1$ than in layer $n$ ) for $k_{\text {eff }}=0.97$, while keeping layers with a reasonable cross section of $130 \times 130 \mathrm{~cm}^{2}$ and width of about $38 \mathrm{~cm}$ (see Fig. 3). Let us briefly discuss the trade-offs involved.

Most of the space is taken by the heavy-water moderator, with a thickness of $35 \mathrm{~cm}$ per layer. The thicker this cell, the better the neutron flux is thermalized, which enhances decoupling between layers. On the other hand, a thicker cell increases the leakage of thermal neutrons into the lead reflector, where they can recouple with previous layers. Taking a larger layer cross section allows the surface leaks in the reflector to be proportionally reduced, and thus, permits an increase in the moderator thickness. This 

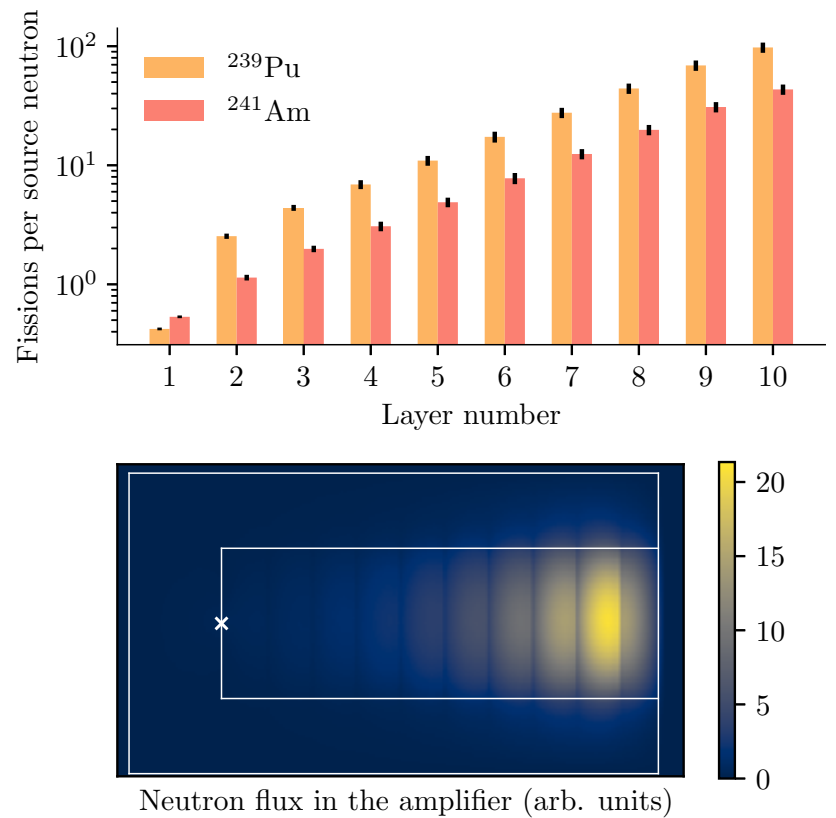

FIG. 3. Neutron amplification for a 10-layer system. After the first layer, the number of fissions per layer is approximately multiplied by 1.6 every layer. Neutron flux also increases geometrically as a function of layer number, and flux near the external source is consequently negligible compared with flux at the end. White cross shows the position of the source.

yields an improved amplification at fixed $k_{\text {eff }}$, at the price of an overall larger amplifier. Using light water would reduce the thickness required to thermalize the neutron flux, but a lower overall amplification is observed because of the higher capture rate.

The mixed-oxide section is chosen to be rather narrow $(1.5 \mathrm{~cm})$ and highly fissile [12] $\left(43 \%{ }^{239} \mathrm{Pu}\right)$. This only weakly exploits the second strategy of fast capture by ${ }^{238} \mathrm{U}$. This is because the backward decoupling effect brought by ${ }^{238} \mathrm{U}$ is outweighed by the increased coupling between layers allowed by a thinner fissile cell. It is still possible to reach some amplification (albeit weaker) by opting for a thicker section with lower ${ }^{239} \mathrm{Pu}$ content (e.g., $8 \%$ ). This then has the advantage of keeping the system subcritical, even in the event of a complete emptying of the moderator (whereas for higher ${ }^{239} \mathrm{Pu}$ contents, the reactivity first decreases after a partial loss of moderator, but increases again after full emptying, as layers recouple backward).

The americium section is taken to have a similar thickness to that of the fissile one $(1.3 \mathrm{~cm})$, and this allows the third strategy to be fully exploited. Finally, the cadmium layer is very thin $(3 \mathrm{~mm})$, which is sufficient to capture most thermal neutrons, while remaining mostly transparent to epithermal and fast ones. This implements the first strategy.
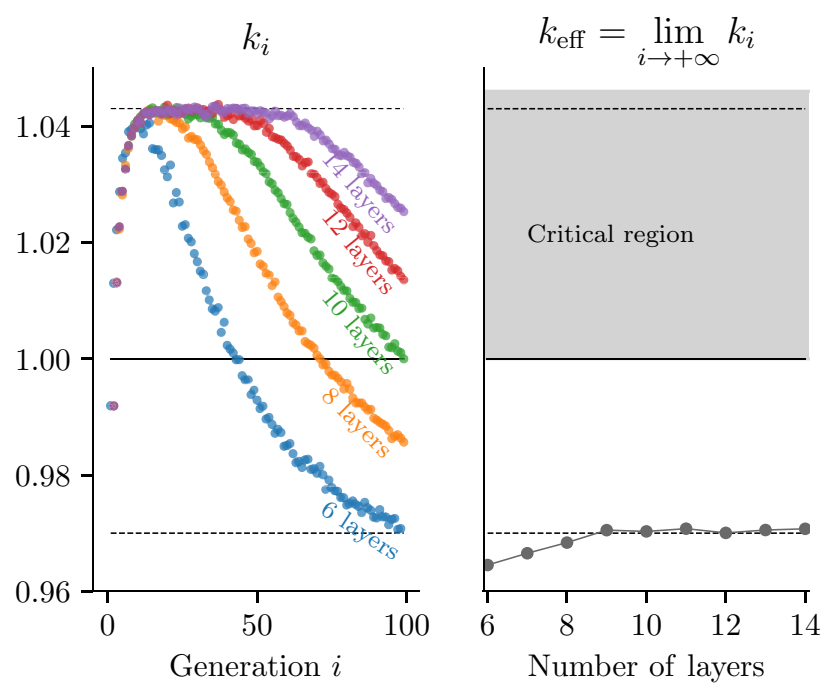

FIG. 4. Transient neutron multiplication. Number of neutrons produced per generation $k_{i}$ (on the left) reaches a plateau at $k \simeq 1.04$, which lasts longer for a higher number of layers. Small initial increase of $k_{\text {eff }}$ as the number of layers is increased (on the right) shows that diodes are not perfect, as expected. However, the system remains subcritical, regardless of the number of layers, with $k_{\text {eff }} \rightarrow 0.97$ for infinitely many layers up to measurement errors.

The way arbitrary geometric amplification is allowed, despite fixed $k_{\text {eff }}$, is transparent in this proposal and follows the argument outlined in Sec. I. Stacking more layers does not bring the system closer to criticality (after a certain point), as backward coupling is suppressed (see Fig. 4). However, the neutron multiplication $k_{i}$ remains larger than 1.0 for a number of generations proportional to the number of layers and passes below 1.0 only once neutrons start to leak at the end of the amplifier. For 10 layers, this happens only after about 100 generations.

\section{DISCUSSION}

With the rudimentary amplifier proposed here, it is possible to reach an amplification as large as one needs. Since the amplification grows exponentially with the number of layers, one can, in principle, have an arbitrarily faint neutron source as the input and even imagine eliminating the accelerator-driven spallation source to use radioisotopes.

Naturally, this comes with severe limitations that are yet to be addressed. Assuming a realistic amplifier could be designed along the lines presented here (including cladding, cooling, control systems, etc.), its safety would still need to be demonstrated. Indeed, the preamplifier operates away from its maximally reactive geometry: a sufficient bending of the layers or melting of the cells could allow the system to become critical. Furthermore, one would need an efficient in situ measurement of $k_{\text {eff: }}$ : in all ADSRs, but even more so in a multilayer system, 
the output power can decrease as $k_{\text {eff }}$ increases, making subcriticality difficult to guarantee in practice. The development of accurate reactivity measurement techniques is an active area of research (see, e.g., Ref. [13,14]). A more minor drawback of a subcritical system with a large preamplification, compared with standard ADSRs, is that the neutron economy is unavoidably worse, as input neutrons are negligible in the overall balance. This means, at least a priori, that thorium breeding in the thermal spectrum is more difficult with such designs.

The preamplifier is studied as a standalone device, and its use to drive a reactor core with a different geometry would necessarily bring some residual backward neutron coupling between the preamplifier and core, slightly increasing the $k_{\text {eff }}$ of the whole. This should be easily verified in fully fledged neutron-transport simulations once a more complete and realistic design is proposed. The quick saturation of $k_{\text {eff }}$ as the number of preamplifying layers is increased (see Fig. 4) suggests that applying the same decoupling strategy and seeing the core merely as a final, much larger, layer should be sufficient to ensure global subcriticality.

In this spirit, an almost trivial and certainly suboptimal example of a reactor core, the basics of which are already understood theoretically, can be mentioned. One may simply consider a stack of the same layers as those of the preamplifier but with a lower ${ }^{239} \mathrm{Pu}$ content to bring the multiplication down to 1.0 (instead of $\simeq 1.6$ ). The fission density would be layer independent in the reactor, and the total power would be linear (instead of exponential) in size. In principle, such a design allows arbitrarily large power for a fixed power density. This ensures that the setup can be cooled and the absorbers and other structures do not decay too quickly from intense neutron flux. However, reducing the power density and neutron flux too much would weaken interest in this type of reactor for actinide burning. Combining high neutron flux, an ability to extract the resulting power, and sufficiently durable absorbers would no doubt be significant challenges for this approach. Constructing the power part of the core (and not just the preamplifier) from so many layers would also likely come with a prohibitive size and complexity. It thus remains important to study more realistic fewlayer power cores synergistic with the present preamplifier proposal.

Aside from problems of implementation and coupling with a power core, there are interesting open questions of a more theoretical nature. In particular, it would be helpful to know what the maximum reachable amplification would be for a given $k_{\text {eff }}$, reactor size budget, and safety constraints. Likewise, what is the lowest $k_{\text {eff }}$ that can be reached with scalable amplification? Multilayer systems also present challenges for accurate Monte-Carlo simulations: naively estimating $k_{\text {eff }}$ and neutron flux everywhere in the system becomes prohibitively costly, as the number of layers is increased, because of the transient exponential gain. Is there a way to do better?

In the end, scalable subcritical amplification presents great challenges but is physically sound. It is, in principle, feasible with materials that exist and with a simple geometry. If realized, such amplifiers could become useful building blocks for fully fledged reactors, to produce electricity, incinerate (some) radioactive waste, or provide on-demand sources for science. As a result, multilayer amplification likely deserves to be studied further and at a finer level of detail. It is hoped that the present paper will stimulate this exploration.

\section{ACKNOWLEDGMENTS}

I am grateful to Sylvain David and Alexis Nuttin for helpful comments and discussions.

[1] There are many other obstacles to a large-scale transmutation strategy for radioactive waste. The improved neutron efficiency of subcritical reactors would only remove a minor obstacle.

[2] H. Nifenecker, O. Meplan, and S. David, Accelerator Driven Subcritical Reactors (CRC Press, Boca Raton, 2003).

[3] L. B. Borst, The convergatron, a neutron amplifier, Phys. Rev. 107, 905 (1957).

[4] F. J. Arias and G. T. Parks, A neutron diode for subcritical multistage multipliers with special reference in tritium breeding, J. Fusion Energy 35, 142 (2016).

[5] V. V. Seliverstov, Multiplication of neutrons from an external source in cascade subcritical systems with unilateral neutron coupling, At. Energy 81, 806 (1996).

[6] G. Kiselev, About some principal possibilities for improvement of electronuclear system's characteristics, Nucl. Eng. Des. 213, 157 (2002).

[7] C.-M. Persson, A. Fokau, I. Serafimovich, V. Bournos, Y. Fokov, C. Routkovskaia, H. Kiyavitskaya, and W. Gudowski, Pulsed neutron source measurements in the subcritical ADS experiment YALINA-Booster, Ann. Nucl. Energy 35, 2357 (2008).

[8] A. Talamo, Y. Gohar, G. Aliberti, Y. Cao, D. Smith, Z. Zhong, H. Kiyavitskaya, V. Bournos, Y. Fokov, C. Routkovskaya, and I. Serafimovich, MCNPX, MONK, and ERANOS analyses of the YALINA Booster subcritical assembly, Nucl. Eng. Des. 241, 1606 (2011), iCONE-17: Heavy Liquid Metal Technologies and Material Challenges for Gen IV Fast Reactors.

[9] V. Becares, D. Villamarin, M. Fernandez-Ordonez, E. Gonzalez-Romero, C. Berglof, V. Bournos, Y. Fokov, S. Mazanik, and I. Serafimovich, Validation of ADS reactivity monitoring techniques in the YALINA-Booster subcritical assembly, Ann. Nucl. Energy 53, 331 (2013).

[10] B. Merk, V. Glivici-Cotruta, and F. Weiß, On the use of different analytical solutions for recalculation of the YALINA-Booster experiment SC3A, Prog. Nucl. Energy 58, 11 (2012). 
[11] P. K. Romano, N. E. Horelik, B. R. Herman, A. G. Nelson, B. Forget, and K. Smith, Openmc: A state-of-the-art monte carlo code for research and development, Ann. Nucl. Energy 82, 90 (2015), Joint International Conference on Supercomputing in Nuclear Applications and Monte Carlo 2013, SNA + MC 2013. Pluri- and Trans-Disciplinarity, Towards New Modeling and Numerical Simulation Paradigms.

[12] Here, isotopically pure ${ }^{239} \mathrm{Pu}$ is used for simplicity, as it provides the highest amplification, but there is no objection to the use of reactor-grade plutonium, which would allow amplification as well.
[13] T. Chevret, J. Lecouey, N. Marie, F. Lecolley, G. Lehaut, G. Ban, A. Billebaud, S. Chabod, X. Doligez, X. Kochetkov, P. Baeten, W. Uyttenhove, G. Vittiglio, J. Wagemans, F. Mellier, V. Bécares, and D. Villamarin, in PHYSOR2014-The Role of Reactor Physics towards a Sustainable Future, 1087437 (Kyoto, Japan, 2014), p. 15.

[14] N. Marie, J. Lecouey, G. Lehaut, T. Chevret, A. Billebaud, S. Chabod, X. Doligez, A. Kochetkov, A. Krasa, F. Lecolley, F. Mellier, W. Uyttenhove, G. Vittiglio, and J. Wagemans, Reactivity monitoring of the accelerator driven VENUS-F subcritical reactor with the "current-toflux" method, Ann. Nucl. Energy 128, 12 (2019). 